\title{
DU MODÈLE D’ALIGNEMENT STRATÉGIQUE DES TI AU LEAPFROG : STRUCTURE CAUSALE OU SYSTÉMIQUE
}

\author{
Author(s) / Auteur(s) : \\ Alphonse - Christian IVINZA LEPAPA \\ Docteur en Sciences de Gestion (ULB, Solvay, 2007) \\ DES en Télématique et Organisation (ULB, Faculté des sciences, 1997) \\ Professeur Honoraire de Gestion de la FWB (Belgique). \\ Professeur MIS et de Télématique des Universités (ESU RDC). \\ Membre Système et Organisation $(S \& O)$ \\ ivinza@hotmail.com
}

\begin{abstract}
Résumé :
Cet article a pour objectif de mettre en avant l'analyse des «Impacts Organisationnels des TIC » qui utilisent les approches systèmes et qui font appel à l'utilisation des modèles. Le centre d'intérêt se situe dans l'école de la conception de systèmes.

Après une brève présentation du modèle d'alignement stratégique (SAM : Strategic Alignement Model, 1991, p.172) de Vankatramam, nous introduisons le concept de Leapfrog.

L'environnement de l'étude concerne les pays en voie de développement (PVD) et les pays sous développé (PSD). Aussi, nous pouvons considérer la RDC et les autres pays africains qui font partie de notre échantillon comme étant des PVD ou des PSD.

La Crise managériale dans les entreprises des PSD et PVD est abordée en deux points: d'abordnous rappelons la classification des pays suivant le potentiel de développement informatique (CIDP - Computer Industrial Development Potential), et ensuite, nous évoquons les facteurs de crise managériale dans ces pays (en Afrique). $L$ 'examen de la résolution des crises managériales des PVD et PSD est effectué sous deux angles. D'abord par un survol du Leapfrog en Afrique: Royaume de l'innovation. Ensuite par l'analyse de l'informatisation des entreprises congolaises suivant le modèle d'alignement stratégique (Ivinza Lepapa, 2007).

Comment les PVD et les PSD adoptent les TIC? Nous repartons d'un échantillon d'une thèse de doctorat réalisée à l'ULB (Ivinza Lepapa et Wilikin Luc, Solvay 2007) nous expliquons et explorons comment l'adoption des TIC dans ces pays ne respectait pas une structure causale linéaire comme dans les pays occidentaux. Mais effectuaient drôlement un bond organisationnel qui migrait vers une structure systémique que les économistes de développement appellent aujourd'hui Leapfrog.
\end{abstract}

Keywords / Mots-clés :

alignement stratégique, TIC, SAM, leapfrog, organisation, crise managériale, modèle, système, causal, PSD, PVD, PD, SISED, SISCA, crise managériale, bond organisationnel, coup stratégique.

Dans cet article, nous présentons d'abord dans la première section le concept d'alignement stratégique (SAM : Strategic Alignement Model). Nous survolons ainsi le cadre du MIT90, le Triangle stratégique français, le cadre théorique de l'alignement stratégique des technologies de l'information (Venkatraman, 1991,1995; Henderson et Venkatraman, 1994, Henderson, Venkatraman and Oldach, 1996) et les différents niveaux de reconfiguration d'entreprises provoquées par les technologies de l'information.

Ensuite, dans la seconde section nous introduisons le concept de Leapfrog (saut de grenouille ou saute mouton ?) après la vision systémique des entreprises.

La troisième section présente la Crise managériale dans les entreprises des PSD et PVD. Deux points sont abordés : la classification des pays suivant le potentiel de développement informatique (CIDP Computer Industrial Development Potential), et les facteurs de crise managériale en Afrique. 
La quatrième section examine la résolution des crises managériales des PVD et PSD,sous deux angles. D'abord par Leapfrog en Afrique : Royaume de l'innovation. Ensuite, par l'analyse de l'informatisation des entreprises congolaises suivant le modèle d'alignement stratégique ${ }^{1}$.

La dernière section aborde l'interprétation des crises managériales dans les entreprises africaines: comme modèle causal ou systémique?

\section{LE MODELE D'ALIGNEMENT STRATEGIQUE DES TI ${ }^{2}$}

Avant de présenter le modèle d'alignement stratégique de Venkatraman, nous rappelons d'abord les grandes lignes du cadre du MIT 90. Celui-ci sera complété par le triangle stratégique de Hubert Tardieu.

\section{Les travaux du MIT 90}

En 1984, à la Sloan Management School du MIT s'est constitué un groupe qui avait pour but d'étudier :

1. la turbulence de l'environnement (social, politique, technique et économique);

2. l'évolution des TI de la prochaine décennie suivant six aspects (matériels-hardware, logiciels-software, réseaux, poste de travail, robotique, puces intelligentes).

Les organisations suivant le paradigme fonctionnaliste des écoles systémiques et de contingence structurelle doivent continuer à survivre devant la turbulence de l'environnement et de l'évolution rapide des TI. Ce qui implique, bien sûr, la maîtrise des changements qui se répercuteront sur les organisations.

Les impacts repérés correspondent à cinq forces de l'organisation qui sont : les technologies, les individus et rôles, les structures, le processus de management et la stratégie. Ces cinq forces sont représentées dans la figure ci-dessous ${ }^{3}$.

\section{Environnement Technologique Externe}

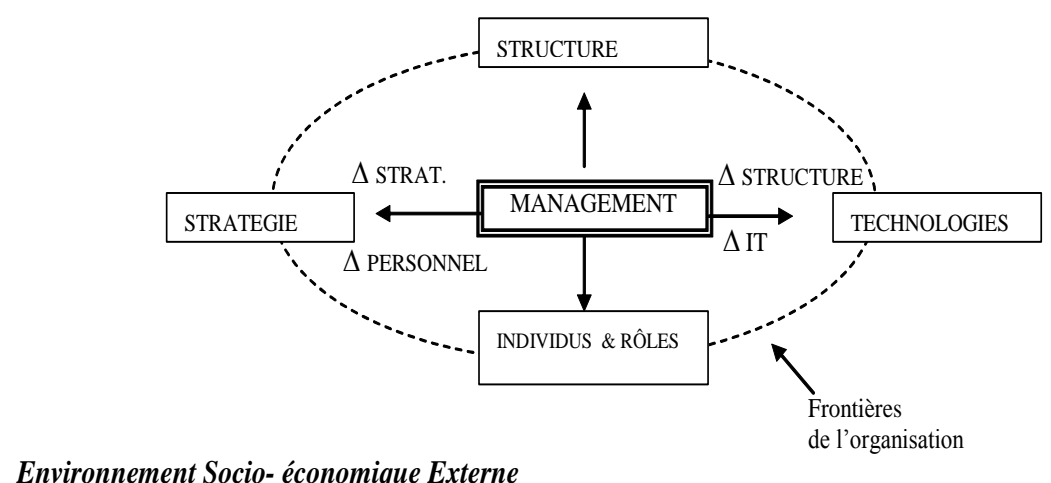

Environnement Socio- économique Externe

Figure 1. Le rôle du management dans le processus du changement (Cadre du MIT 90)

\footnotetext{
1 IVINZA LEPAPA, Analyse de l'introduction de l'EDI dans des entreprises congolaise : contribution à impact organisationnel des TI, Tome II, Solvay Business School, Bruxelles, 2007, pages $432-435$.

2 SCOTT MORTON Michael S.(editor), The Corporation of the 1990's, Information technology and Organization transformation, New York, Oxford University Press, 1991 ; pour l'édition française L'entreprise compétitive au futur : Technologies de l'information et transformation de l'organisation, Les éditions d'organisations, Paris, 1995, pages 3 à 28. 3 SCOTT MORTON Michael S., op.cit., 1991, 1995, page 35.
} 


\section{Le triangle stratégique}

A la suite des travaux du MIT 90, Hubert Tardieu et Bernard Guthmann ${ }^{4}$ présentent le fondement du triangle stratégique, qui est un système d'auto - référence partielle comprenant la stratégie, la structure et les technologies de l'information. ".Le triangle stratégique propose trois composants (TI, structure, stratégie) qui sont articulés autour du système d'information stratégique (SIS).

"La stratégie détermine les structures de l'entreprise, mais les technologies de l'information aident à déterminer la stratégie et à en faire le suivi. Les structures déterminent l'usage des technologies de l'information et permettent de déterminer les coups stratégiques qui eux-mêmes constituent la stratégie." ${ }^{5}$ Cette notion de système auto - référence était déjà évoquée dans l'article Système d'information et pilotage de l'entreprise : "quelques boucles étranges". ${ }^{6}$

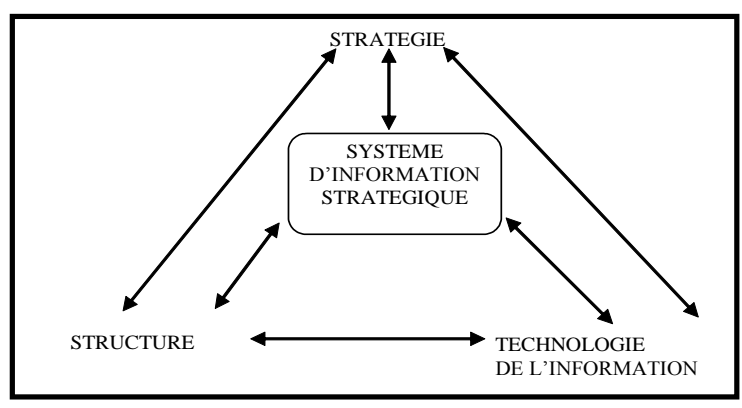

Figure 2. Le Triangle stratégique

\section{Cadre théorique de l'alignement stratégique des technologies de l'information (Venkatraman, 1991,1995; Henderson et Venkatraman, 1994, Henderson, Venkatraman and Oldach, 1996)}

Dans une de ses conclusions, le rapport du MIT 90 a montré que dans l'environnement concurrentiel actuel, aucune entreprise ne peut se passer des TI. Pour fonctionner dans cet environnement, les entreprises doivent aligner consciemment leurs stratégies et leurs structures sur les TI. Cet alignement doit être dynamique, c'est à dire évolutif entre le contexte stratégique de l'entreprise et des TI dans le cadre d'un modèle appelé SAM (Strategic Alignement Model). ${ }^{7}$

Le modèle théorique d'alignement stratégique (SAM) proposé par Venkatraman comprend quatre composants : la stratégie commerciale (stratégie d'entreprise), l'infrastructure et les processus de l'entreprise (infrastructure organisationnelle), la stratégie des TI, l'infrastructure et les processus des TI (infrastructure des SI).

\footnotetext{
4 TARDIEU Hubert et GUTHMANN Bernard, Le Triangle Stratégique : Stratégie, Structure et Technologie de l'information, Edition de l'organisation, Paris, 1992.

5 TARDIEU Hubert et GUTHMANN Bernard, op.cit., 1992, page 67.

6 TARDIEU Hubert et THEYS Michel, Systèmes d'information et pilotage de l'entreprise : quelques boucles étranges" in Revue Internationale du systémique, numéro spécial "L'entreprise système", Volume 1, n 4, 1987.

7 VENKATRAMAN N., IT - Induced Business Reconfiguration in M.S. Scott Morton The Corporation of the 1990's, op. cit., 1991, pages 122-158.
} 


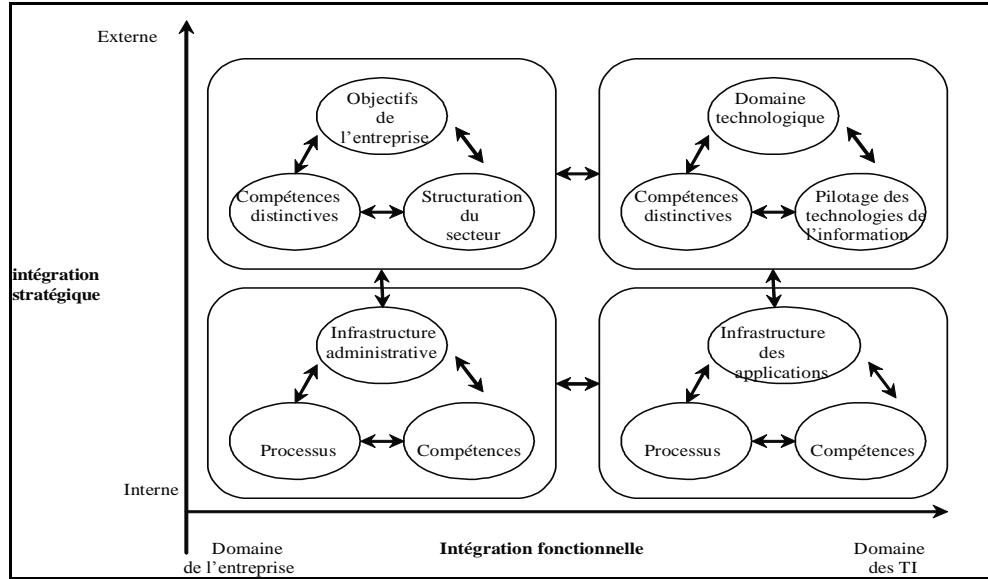

Figure 3. Le modèle d'alignement stratégique

Ce modèle propose trois types de relation :

- bivariée verticale qui représente l'intégration stratégique (stratégie d'entrepriseinfrastructure organisationnelle, stratégie des TI- infrastructure des TI),

- bivariée horizontale qui représente l'intégration fonctionnelle (stratégie d'entreprisestratégie des TI, infrastructure organisationnelle- infrastructure des TI),

- multivariée qui donne les quatre triades du schéma d'alignement stratégique : potentiel concurrentiel (stratégie d'entreprise- infrastructure des SI - infrastructure organisationnelle), potentiel technologique (stratégie d'entreprise- stratégie des TI infrastructure des SI) valeur de l'entreprise (stratégie d'entreprise- stratégie des TI infrastructure organisationnelle), niveau de service (stratégie d'entreprise- stratégie des TI -infrastructure des SI).

\section{Les niveaux de reconfiguration d'entreprises provoquées par les technologies de l'information. ${ }^{8}$}

Ce modèle propose cinq niveaux de reconfiguration provoqués par les TI : (1) Exploitation en local, (2) Intégration interne, (3) Reconception des processus de gestion, (4) Reconception du réseau de gestion, (5) Redéfinition des objectifs de l'entreprise.

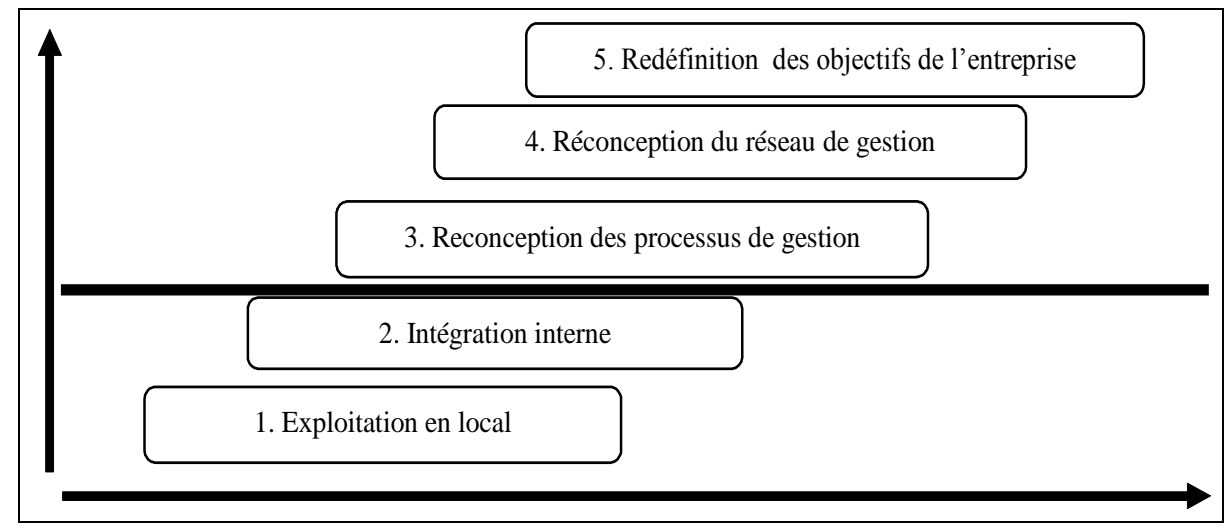

Figure 4. Les cinq niveaux de reconfiguration provoqués par les technologies de l'information

8 Ce paragraphe s'inspire complètement de VENKATRAMAN N., IT - Induced Business Reconfiguration in M.S. Scott Morton The Corporation of the 1990's, op. cit., 1991, pages 122-158. 


\section{$\underline{1^{\circ} \text { Le niveau } 1 \text { : Exploitation en local. }}$}

Il concerne l'utilisation des technologies de l'information dans le cadre d'une fonction de l'entreprise telle que la production ou le commercial ce qui implique la mise en œuvre d'applications des technologies de l'information destinées améliorer l'efficacité des opérations.

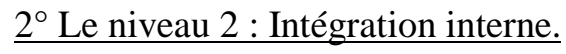

C'est une extension logique du niveau précédent en ce sens que les possibilités des technologies de l'information sont exploitées dans toutes les fonctions de l'entreprise où cela est faisable.

Deux types d'intégration sont possibles :

- l'intégration technique : c'est l'intégration des différents systèmes d'applications utilisant une plate-forme informatique commune,

- l'intégration organisationnelle des différents rôles et responsabilités exploitant les possibilités d'intégrations techniques.

En d'autres termes, la mise en ouvre d'une plate-forme informatique commune sert à intégrer les activités de gestion de l'organisation; ce qui doit en améliorer potentiellement l'efficacité.

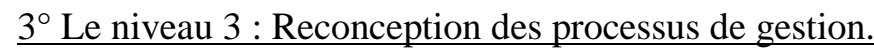

Elle concerne la reconfiguration de la gestion en utilisant les technologies de l'informatique comme levier central. Au lieu de traiter les processus de gestion existants comme une contrainte dans la définition d'une infrastructure informatique optimale, c'est le processus de gestion lui-même qui est réétudié pour exploiter au mieux les possibilités offertes par les technologies de l'information. Ce qui est un reflet d'efforts conscients de création d'une homogénéité.

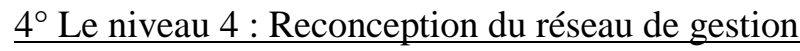

Elle consiste à se servir des technologies de l'information pour mettre en place un système d'information qui va utiliser les fournisseurs, clients et autres tiers capables de contribuer à l'objectif de la firme. En d'autres termes, il s'agit de quitter l'organisation traditionnelle pour aller vers une organisation «virtuelle» ou en «réseau» qui permettra d'atteindre un objectif particulier.

L'intégration électronique des partenaires externes devient ainsi le problème dominant du management stratégique.

$\underline{5^{\circ} \text { Le niveau } 5 \text { : Redéfinition des objectifs de l'entreprise }}$

A ce niveau, l'organisation décide de rompre avec le passé et décide d'exploiter les nouvelles technologies en terme de marchés ou de produits.

\section{$\underline{6^{\circ} \text { Conclusion }}$}

\section{Les niveaux 1 et 2 sont évolutifs, les niveaux 3 à 5 sont révolutionnaires.}

Les deux premiers niveaux ont un caractère évolutif et se contentent de modifications relativement incrémentales des processus existants.

En revanche, les trois autres niveaux sont conceptualisés comme quelque chose de révolutionnaire et ils exigent des changements de fond dans la nature même du processus de gestion. 


\section{ET VINT LEAPFROG}

Avant de parler du concept de Leapfrog (saut de grenouille ou saute mouton ?), nous synthétisons d'abord la vision systémique des entreprises.

\section{Vision systémique des entreprises}

Les entreprises et les organisations sont considérées, selon les écoles systémique et de la contingence du paradigme fonctionnaliste, comme un système en interaction avec son environnement. Seguin et Chanlat (1983, p. 26) définissent «l'organisation comme un système qui importe certains éléments de son environnement, les transforme et les exporte vers l'environnement» ${ }^{9}$. D'autre part, Luc Wilkin (1999-2000, p. 180) considère que «les organisations sont des systèmes ouverts, dont le mode de fonctionnement interne doit être adapté aux caractéristiques de l'environnement $\rangle^{10}$. Donc, pour qu'une organisation soit en interaction avec son environnement, il faut qu'elle soit considérée comme un système ouvert, celui pour lequel la survie dépend de l'extérieur.

C'est pourquoi, les entreprises fonctionnement suivant le modèle de l'école de la contingence «Contexte - Structure - Performance ». Les mutations insufflées par la recherche des performances et l'évolution rapide des technologies dans tous les domaines poussent les entreprises à adopter une structure organisationnelle répondant aux nouveaux défis de l'organisation. Ces changements organisationnels conduisent à des (NFO) Nouvelles formes d'organisation : les entreprises virtuelles, les entreprises réseaux, le travail à distance, la gestion de la chaîne de valeur, etc.

Dans la recherche de la performance, chaque firme cherche sa survie dans son environnement. La théorie des systèmes « est la base sur laquelle s'appuient les théoriciens de la contingence, ainsi que sur une vision structuro- fonctionnaliste pour qui la survie des organisations dépend de leur adaptation à l'environnement $»^{11}$.

L'analyse systémique implique nécessairement l'option d'un holisme méthodologique qui comporte une causalité externe (explicative) et une causalité interne (compréhensive). La causalité externe (explicative) expliquera les diverses connexions, alors que la causalité interne (compréhensive) donnera la signification des phénomènes.

\section{Leapfrog : saut de grenouille ou saute mouton ?}

\section{$1{ }^{\circ}$ Leapfrog dans les pays en développement.}

Le concept de «saut en avant, saut de grenouille ou saute mouton» est une théorie qui propose le développement des pays en évitant les technologies et industries intermédiaires. Ce concept de sautemouton se réfère à des pays sous développés (PSD) et en développement (PVD).

La téléphonie sans fil est un exemple de ce développement technologique et industriel «sautemouton»: les PSD et les PVD ont passé directement aux réseaux non filaires (réseau satellite et réseau mobile), sans avoir expérimenté les réseaux filaires (PSTN, CSPDN, PSPDN et même RNIS), surtout avec l'apparition O3B.

\section{$\underline{2^{\circ} \text { Tunneling }}$}

Un concept étroitement lié au leapfrog est celui du «tunneling» à travers la courbe environnementale de Kuznets (EKC). ${ }^{12}$ Ce concept «propose que les pays en développement puissent tirer des enseignements des expériences des pays industrialisés et restructurer leur croissance et leur

\footnotetext{
${ }^{9}$ SEGUIN F. et J.F. CHANLAT J.F. (1983), L'analyse des organisations, une anthologie sociologique, Tome I Les théories de l'organisation, Gaëtan Morin éditeur, Paris, 1983, p. 26.

${ }^{10}$ WILKIN Luc, (1999) Principes généraux d'organisation et de gestion, 2ième édition, PUB, année académique 1999-2000, p. 180.

${ }_{11}$ GUITIERREZ Luiz Homero (1993), Les transformations de la fonction du personnel. La contribution de l'analyse contextualiste. UCL, ESPO, Thèse doctorale ${ }^{\circ} 226$ (nouvelle série), IST Novembre 1993, page 47, Louvain La Neuve.

12 MUNASINGHE, M. (1999). "La dégradation de l'environnement est-elle une conséquence inévitable de la croissance économique: les tunnels à travers la courbe environnementale de Kuznets".in Économie écologique . 29 (1): 89-109. doi : 10.1016 / S0921-8009 (98) 00062-7.
} 
développement afin de s'attaquer rapidement aux dommages environnementaux irréversibles et de les «canaliser» à travers toute EKC potentielle ». ${ }^{13}$

Les écologistes considèrent que le concept de leapfrog permet aux PSD et PVD d'adopter des technologies plus vertes que les pays en développement (PD) qui utilisent des anciennes technologies polluantes. Alors que du point de vue économique, nous pensons que les PD sont devant un problème de choix économique désinvestir ou pas?

De même, les PVD à notre avis profitent plus du progrès technologique des PD pour sauter les technologies qi produisent des produits marché «vache à lait», ceux qui génèrent des grandes liquidités avec peu de financement.

\section{CRISE MANAGERIALE DANS LES ENTREPRISES DES PSD ET PVD}

Cette section présente la Crise managériale dans les entreprises des PSD et PVD. Deux points sont abordés: la classification des pays suivant le potentiel de développement informatique (CIDP Computer Industrial Development Potential), et les facteurs de crise managériale en Afrique.

\section{Classification des pays suivant le potentiel de développement informatique (CIDP - Computer Industrial Development Potential)}

Nous référant à la classification du niveau de développement économique des pays utilisée par Palvia et alii qui ont analysé les différents thèmes de recherche d'impact des TI par régions et pays, nous pouvons considérer les pays africains comme des pays en développement ou sous développés.

Ce que confirme d'ailleurs Del Castillo dans une étude de l'OCDE (2003, p.1) ${ }^{14}$ Cette étude classe le développement économique des pays en quatre catégories :

- pays développé,

- pays nouvellement industrialisé,

- pays en développement

- et pays sous développé ${ }^{15}$.

Cette classification utilisée par Palvia et alii est inspirée par celle des Nations Unies (Porat, 1977 ; Kalman, 1979) qui classe les pays suivant leur potentiel de développement informatique (CIDP Computer Industrial Development Potential) :

- pays à potentiel informatique avancé,

- pays à potentiel informatique opérationnel,

- pays à potentiel informatique essentiel

- et pays à potentiel informatique embryonnaire.

Les pays africains peuvent être considérés comme étant des pays à potentiel informatique essentiel et embryonnaire.

\section{Facteurs à l'origine des crises managériales des entreprises des PSD et PVD}

Les facteurs sont examinés du point de vue systémique (concept d'homéostasie) et du point de vue managérial (le changement organisationnel).

$1{ }^{\circ}$ Concept d'homéostasie

Le changement organisationnel s'explique aisément par le concept d'homéostasie, suivant

l'hypothèse d'Edgar Morin qui considère que la vie d'un système implique un double mouvement : ${ }^{16}$

- un mouvement de corruption et de désorganisation,

- un mouvement de fabrication et de réorganisation.

\footnotetext{
${ }^{13}$ Wikipédia consulté 6 septembre 2018.

${ }^{14}$ Del Castillo, OCDE, 2003, p.1.

15 PALVIA Prashant C., PALVIA Shailendra C. Jain and WITHWORTH (2002), Global Information Technology : a meta analysis of Key Issues in Information \& Management, Elsevier $\mathrm{n}^{\circ} 39$ pages 403-414.

${ }^{16}$ Cette partie résume Edgar Morin, 1977, pages 182 à 223 citée par BOQUE Jean-Michel, L'organisation en tant que système paradoxal, in BONAMI Michel et alii, Management des systèmes complexes : «Pensée systémique et intervention dans les organisations », 1993, de Boeck page 15 .
} 
On peut donc, considérer l'homéostasie comme «la conjonction des processus par lesquels un système résiste au courant général de corruption et de dégénérescence. Elle désigne donc l'ensemble des rétroactions correctrices et régulatrices par lequel la dégradation déclenche la production et la réorganisation.». ${ }^{17}$

$2^{\circ}$ Le changement dans l'environnement des entreprises

Le concept systémique d'homéostasie nous apprend que l'organisation évolue dans un environnement qui offre des opportunités d'une part et des menaces d'autre part. «De fait, l'entreprise est un organisme vivant qui réagit aux changements et cherche à en tirer parti pour s'assurer un avantage sur ses concurrents. ${ }^{18}$

Parmi les facteurs qui poussent les organisations à changer, on peut citer :

- «l'accroissement des connaissances,

- les progrès techniques,

- l'évolution des systèmes des valeurs,

- et l'internationalisation des économies.

La combinaison de ces facteurs crée un environnement de plus en plus concurrentiel et changeant autour de l'entreprise, l'obligeant à des efforts d'innovation de plus en plus intenses. $»^{19}$

\section{RESOLUTION DES CRISES MANAGERIALES DES PVD ET PSD}

Cette section examine la résolution des crises managériales des PVD et PSD, sous deux angles. D'abord par Leapfrog en Afrique: Royaume de l'innovation. Ensuite, par l'informatisation des entreprises congolaises suivant le modèle d'alignement stratégique (Venkatraman, 1991, p.172).

\section{Leapfrog en Afrique : Royaume de l'innovation}

$1^{\circ}$ Cadre théorique : l'avantage de l'arriération

Dans un article publié dans la tribune du 20 octobre 2017, Makhtar Diop, vice-président de la Banque mondiale pour l'Afrique se pose la question suivante. L'Afrique peut-elle sauter des étapes pour accélérer son développement et s'imposer comme le royaume de l'innovation ?20 Pour lui, «La Banque mondiale a un rôle à jouer dans ce processus : nous atténuons le risque pays à l'aide de différents outils, tels que les garanties, ce qui permet d'attirer des investissements internationaux de qualité et des financements locaux. Toutes les tentatives n'aboutiront pas. Les pays africains, le secteur privé et les partenaires de développement doivent être prêts à prendre des risques et à apprendre de leurs échecs. » ${ }^{21}$

Plusieurs cas de leapfrog sont relevés en Afrique.

Le Kenya est aujourd'hui un des leaders mondiaux de la banque mobile, grâce au M-Pesa, qui est un service de paiement par téléphone portable

«En Afrique subsaharienne, le téléphone portable fait désormais office de banque pour des millions de personnes qui n'avaient aucun espoir de pouvoir un jour ouvrir un compte bancaire traditionnel. » ${ }^{22}$

«Au Cameroun, le Cardiopad permet de faire des examens de type électrocardiogramme à distance. Commercialisé en 2014, l'appareil s'exporte désormais dans de nombreux pays, dont l'Inde et le Canada. $»^{23}$

\footnotetext{
${ }^{17}$ BOQUE Jean-Michel, L'organisation en tant que système paradoxal, in BONAMI Michel et alii op.cit., 1993, page 76.

${ }^{18}$ JABES Jak, Changement et développement organisationnel, in AUBERT Nicole et alii, Management « Aspects humains et organisationnels », PUF fondamental, 1996, Paris, page 594.

${ }^{19}$ Ibidem.

${ }^{20}$ MAKHTAR Diop, «L'Afrique, futur royaume de l'innovation ?» in Tribune du 20 octobre 2017.

${ }^{21}$ MAKHTAR Diop, op.cit.

${ }^{22}$ MAKHTAR Diop, op. cit.

${ }^{23}$ GRAS Stéphan-Eloïse et BABINET Gilles, Numérique : le gigantesque saut de grenouille africain, in Tribune : Les innovations venues d'Afrique n'ont pas fini de nous étonner ! Plongée au coeur du "leapfrogging" africain avec les fondateurs d'Africa4tech. Publié le 26/10/2016 à 16:06 - Modifié le 26/10/2016 à 19:34 | Le Point Afrique
} 
Selon GRAS Stéphan-Eloïse et BABINET Gilles, ce qui est fascinant est le fait que l'économiste Alexander Gerschenkron appelait « l'avantage de l'arriération ». Cette expression, signifie que «le fait d'avoir pris du retard permet de sauter certaines étapes et d'adopter immédiatement des technologies plus avancées, souvent développées sur place». 24

«Or, en accédant aux réseaux 4G pour ainsi dire simultanément aux Occidentaux, en disposant de services numériques qui n'existent pas même en Occident, les Africains ont clairement démontré qu'ils effectuaient un «saut de grenouille $» .{ }^{25}$

\section{$2^{\circ}$ Génération Internet et du Millénaire en Afrique et au Congo}

Ces innovations ont trouvé un bon cadre de développement humain : l'existence des générations Internet et du millénaire en Afrique.

Les Africains et les congolais actuels sont en majorité, issus de ces deux générations, ils vivent l'ère du numérique (ordinateur, baladeur, Gsm) et devront être parmi les plus instruits de ce continent.

- Ils sont nés dans la société du savoir comme les autres enfants du monde,

- Ils sont marqués par l'internet comme les autres enfants du monde.

En raison de la mondialisation des Technologies de l'Information et de la communication (TIC), qui empêcherait l'usage des nouvelles technologies dans les milieux africains ?

$\underline{3^{\circ} \text { Problématique de l'influence des générations Internet et du Millénaire sur les innovations }}$ technologiques

Les natifs des générations Internet et du Millénaire sont très indépendants et autonomes. Ce trait de caractère provient du fait qu'ils sont des chercheurs actifs d'informations et non pas des capteurs passifs. "Leur accès sans précédent à l'information leur donne aussi la capacité d'acquérir le savoir nécessaire » pour acquérir et mettre en œuvre des nouvelles technologies.

Le fait que ces deux générations soient indépendants, autonomes et cherchent les sensations fortes, ils préfigurent une nouvelle race d'africains capable de mettre en œuvre des nouvelles techniques et industries.

Les TIC font évoluer ces natifs « non pas vers l'exclusion, mais vers une plus grande inclusion sociale. » Internet pousse ces africains à passer de la sphère locale à la sphère mondiale. Dans ces conditions, il est difficile d'empêcher ces générations d'acquérir les nouvelles technologies en même temps que leur collègue d'autres continents, car leur migration de l'environnement local vers l'environnement mondial est irréversible.

\section{L'informatisation des entreprises congolaises suivant le modèle d'alignement stratégique (Venkatraman, 1991, p.172)}

Ces résultats proviennent d'une recherche effectuée à la Solvay Business School de l'ULB. ${ }^{26}$

La reconfiguration de Venkatraman comprend 5 niveaux : exploitation en local (1), intégration interne (2), reconception de processus de gestion - business process redesign (3), reconception du réseau de gestion business network redesign (4), redéfinition des objectifs de l'entreprise (5).

\section{$1^{\circ}$ Echantillon 1 : EDI intra - entreprise}

Suivant le modèle de Venkatraman (1991), ces entreprises congolaises oscillent entre les étapes de "l'exploitation en local » et de "l'intégration interne ». Les entreprises avancées de cette catégorie s'arrêtent à la seconde étape de reconfiguration « intégration interne ».

L'utilisation des coups stratégiques (lien entre la stratégie et l'informatique) est une boîte noire pour les entreprises congolaises dont le besoin d'échange de données est limité entre leurs propres

\footnotetext{
${ }^{24}$ GRAS Stéphan-Eloïse et BABINET Gilles, op.cit.

${ }^{25}$ GRAS Stéphan-Eloïse et BABINET Gilles, op. cit.

26 IVINZA LEPAPA, Analyse de l'introduction de l'EDI dans des entreprises congolaise: contribution à impact organisationnel des TI, Tome II, Solvay Business School, Bruxelles, 2007, pages 432 - 435.
} 
unités. Ce qui correspond au rejet des systèmes d'information stratégique dans la première étape (exploitation en local) de reconfiguration d'entreprises provoquées par les TI. Dans cette catégorie, les entreprises ne sont pas alignées stratégiquement aux TI.

A la question de savoir comment les stratégies de base se répartissent dans les coups stratégiques. On constate que plusieurs entreprises de cette catégorie ignorent la notion de coup stratégique. Les quelques utilisations recensées concernent le besoin d'intégration externe à un marché ou à une hiérarchie électronique. Cette intégration ne se faisait souvent pas pour gagner un avantage concurrentiel, mais plutôt pour s'adapter au changement des propriétés contextuelles de la communauté dans laquelle ces entreprises appartiennent. Les SIS (système d'information stratégique) utilisés sont des SISED (strategic information system for economic development.) propre à un pays en développement ou au PSD. ${ }^{27}$

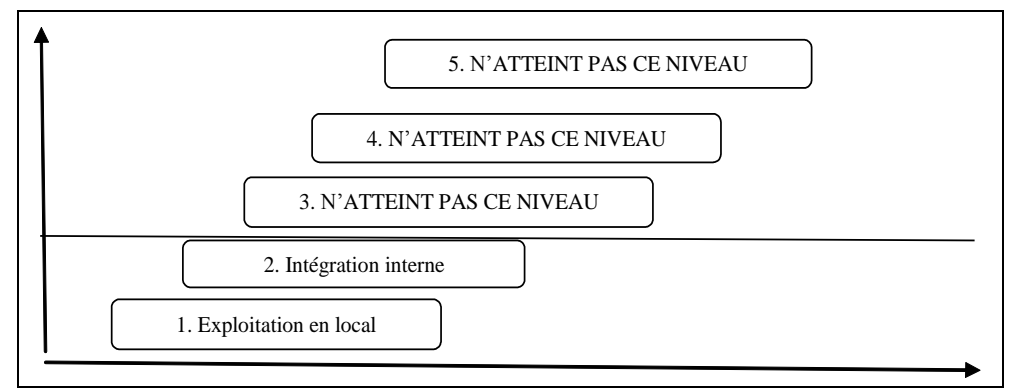

Figure5. Reconfiguration provoquée par les TIC suivant une structure causale linéaire

\section{$\underline{2^{\circ} \text { Echantillon 2 : EDI extra - entreprise }}$}

Les entreprises de cette catégorie effectuent un «bond organisationnel » pour atteindre le niveau révolutionnaire de reconfiguration d'entreprise en passant sans transition de l'étape 1 «exploitation en local» à l'étape 4 « Business Network Redesign ».

Pour l'EDI extra entreprise (catégorie 2), le besoin d'intégration externe conduit à la mise en œuvre des SISED et des SISCA- strategic information system for competitive advantage (Palvia, Palvia et Zigli; 1990), ainsi qu'à l'organisation des marchés et des hiérarchies électroniques (Malone, Yates et Benjamin ; 1994). On assiste au passage de ces entreprises à la quatrième étape de la reconfiguration des entreprises «Business Network Redesign» (Venkatraman, 1991) qui conduit à l'implantation des organisations virtuelles supportées par les TI sous forme d'entreprise réseau (Libé et Paré, 1999, p. 18).

Devant le phénomène de guichet unique, plusieurs entreprises sont appelées à utiliser des coups stratégiques pour permettre l'EDI avec les partenaires. A ce stade, les entreprises effectuent un «bond organisationnel» pour atteindre le niveau révolutionnaire de reconfiguration d'entreprise en passant sans transition de l'étape 1 «exploitation en local» à l'étape 4 «Business Network Redesign » (Venkatraman, 1991, p.172). Les entreprises de cette catégorie n'ont pas un souci d'intégration interne et de contrôle des succursales, mais plutôt le besoin d'intégration externe découlant des rapports de l'organisation et son environnement extérieur.

L'intégration informatique de ces entreprises ne suit pas le schéma de Baile, c'est-à-dire n'évolue pas de l'intégration interne vers l'intégration externe (1997b, p.169).

On assiste à l'apparition des SIS de type SISED qui préfigure l'ère de l'organisation virtuelle supportée par les TI (Libé et Paré, 1999, p.14-22) dans les entreprises des PSD. L'apparition des coups stratégiques provient des besoins d'intégration externe par des hiérarchies et des

27 PALVIA Prashant, PALVIA Shailendra and ZIGLI Ronald M.(1990), Models and requirements for using Strategic Information systems in Developing nations, in International Journal of Information Management, volume 10, issue 2, June 1990, pages 117-126. 
marchés électroniques par les SISCA (c'est le cas de la BCCE et de la DGRAD) et SISED (c'est le cas de l'OCC et de l'OFIDA et de l'ONATRA).

Dans cette catégorie, même n'utilisant pas la stratégie les entreprises sont obligées de s'aligner en utilisant le SISED.

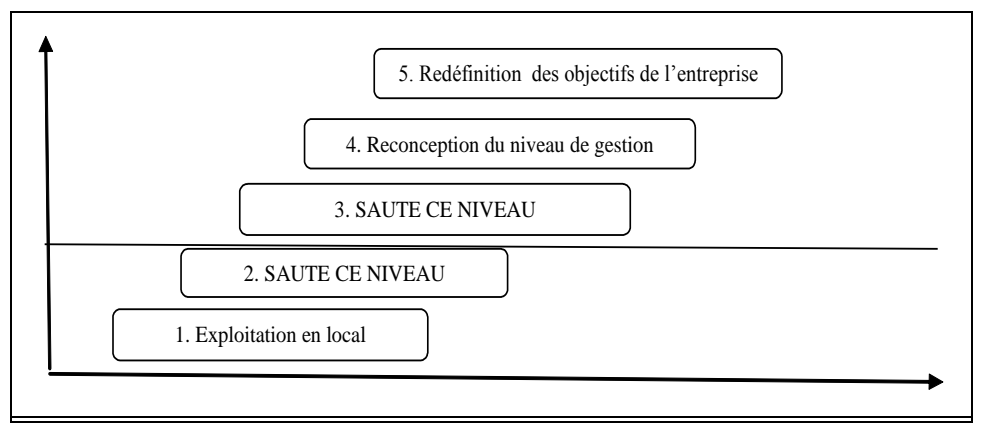

Figure 6. Reconfiguration provoquée par les TIC suivant une structure systémique par bond organisationnel (saut)

\section{INTERPRETATION DES CRISES MANAGERIALES DANS LES ENTREPRISES CONGOLAISES: MODELE CAUSAL OU SYSTEMIQUE ?}

Les entreprises congolaises se répartissent principalement sur trois des cinq niveaux de l'alignement stratégique : exploitation en local (1), intégration interne (2), reconception du réseau de gestion (4).

La théorie des systèmes qui est la base sur laquelle nous fondons notre interprétation considère la survie des organisations dépend de leur adaptation à l'environnement dans lequel, elles se situent.

Nous allons situer notre interprétation sur les deux échantillons précédents.

$\underline{1^{\circ} \text { Echantillon } 1 \text { : EDI intra - entreprise : structure causale linéaire }}$

Les entreprises de cette catégorie adoptent le TIC et s'organise de façon linéaire. Elles vont du niveau 1 au niveau 2. Ignorant de l'usage des stratégies dans les TIC, elles n'évoluent pas vers les niveaux supérieurs.

Les organisations respectent une structure linéaire causale. Les TIC influencent l'organisation et son système d'information de ces entreprises. La propriété principale pour maintenir la survie des entreprises de cette catégorie est l'auto-apprentissage.

«L'apprentissage exige en effet que la relation entrée-sortie ne soit ni trop lâche (pour permettre une réponse adaptée au stimulus), ni trop rigide (pour pouvoir modifier cette réponse si elle est inadéquate)». ${ }^{28}$

Le fait que la stratégie des entreprises congolaises subisse des transformations réversibles permet aux managers d'adapter leur comportement face aux convergences et aux divergences. Ces transformations conduisent soit à l'implosion, soit à l'explosion de l'organisation mettant en péril les principes qui permettent la survie de cette organisation.

\section{$\underline{2^{\circ} \text { Echantillon 2: EDI extra - entreprise structure systémique }}$}

Dans cette catégorie, certaines entreprises pour assurer leur survie dans leur environnement de travail commencent à utiliser des systèmes d'information stratégique (SIS) et des coups stratégiques (lien entre la stratégie et l'informatique). Ces entreprises effectuent un bond organisationnel, elles sautent de niveau 1 au niveau 4.

L'adoption des TIC s'apparente à un système critique auto - organisé qui est faiblement chaotique. Les systèmes critiques auto - organisés «évoluent vers un état critique tel qu'une petite perturbation déclenche une réaction en chaîne qui touche un nombre aléatoire d'éléments du système. Selon le nombre d'éléments touchés, la perturbation provoque un événement 
mineur ou une catastrophe $» .{ }^{29}$ Les entreprises de cette catégorie doivent obligatoirement chercher l'équilibre pour assurer leur survie, autrement elles sont vouées à disparaitre.

Le cas d'auto -organisation le plus perceptible est celui des entreprises du guichet unique qui ont adopté les SISED (strategic information system for economic development) et une organisation réseau WAN Extranet sans les avoir prévu.

«Un système peut ainsi connaître des phases de déstructuration et de restructuration se recouvrant partiellement. (...) L'auto -organisation peut intervenir lors de modifications lentes et continues de l'environnement du système.(...) Mais l'auto -organisation intervient également lors de modifications brutales. $»^{30}$

\section{CONCLUSION}

L'alignement stratégique des entreprises de la catégorie 1 ressemble à une structure causale linéaire. Dans ce modèle les TIC sont des variables indépendantes qui influencent l'organisation de l'entreprise (structure organisationnelle et système d'information).

Tandis que dans la catégorie 2, les entreprises effectuent des bonds organisationnels et passent, selon le modèle de Vankatraman, sans transition du niveau 1 au niveau 4 sans transition. L'alignement stratégique ressemble à un modèle systémique où les variables peuvent changer de rôle à tout moment (indépendante ou dépendante), les TIC, la stratégie peuvent être seuls ou de facon conjoint des variables indépendantes ou dépendantes.

Ces entreprises effectuent des bonds organisationnels que les économistes de développement appellent leapfrog (saut de grenouille ou saute mouton). Ces bonds organisationnels ont pour effet un saut technologique, qui offre ce que Gras et Babinet appellent l'avantage de l'arriération.

\section{REFERENCES}

BOWONDER B., MIYQAKE T. and SINGH T. Monish (1993), Emerging trends in information technology: Implications for developing countries, in International Journal of Information Management, volume 13, issue, June 1993, pages 183-204.

DAVIS Gordon B., (1992) A Model for Adoption and Diffusion of Information Systems in Less Developed Countries, in PALVIA Shailendra C. Jain , PALVIA Prashant C. and ZIGLI Ronald M., (1992) The Global Issues of Information Technology Management, Idea Group Publishing, Harrisburg.

DE BOER Sirp J. and WALBEEK Mirjam M., Information Technology in developing countries : A studies to guide policy formulation, in International Journal of Information Management, volume 19, 1999, pages 207-218.

DUBE Line et PARE Guy, Les Technologies de l'Information et l'organisation à l'ère du virtuel employés, in Revue internationale de Gestion, volume 24, numéro 2, Eté 1999, pages 14-22

DUPUY Y. et alii, op.cit., 1989, page 250 et sq ; LESCA Humbert, Système d'information pour le management stratégique de l'entreprise «L'entreprise intelligente», McGrawHill, Paris, 1986, pages $112-122$.

JACKSON Carey-Ann et EKSTEEN Johan, Création de contenu local et commerce électronique : point de vue sud-africain page 201 in GOLDSTEIN Andrea et O'CONNOR David (éditeur), Commerce électronique et Développement, Etudes du centre de développement, OCDE, 2002.

29 TREMBLAY Christian, op.cit., 1992, page 132.

30 WALLISER Bernard, op.cit., Paris, 1977, page 87. 
JANCZEWSKI LECH J, Factors of information Technology Implantation in Under-Developed countries: Example of the West Africa Nations, 1992, page 187-212 in PALVIA, PALVIA and ZIGLI, op. cit., 1992.

GRAS Stéphan-Eloïse et BABINET Gilles, Numérique : le gigantesque saut de grenouille africain, in Tribune: Les innovations venues d'Afrique n'ont pas fini de nous étonner ! Plongée au coeur du "leapfrogging" africain avec les fondateurs d'Africa4tech. Publié le 26/10/2016 à 16:06 - Modifié le 26/10/2016 à 19:34 | Le Point Afrique

IVINZA LEPAPA Alphonse, " Informatique Stratégique : Cadre théorique et Applications dans les entreprises congolaises ", Bookelis, Paris, 2016.

IVINZA LEPAPA Alphonse, " Analyse de l'introduction de l'EDI dans les entreprisescongolaises : une contribution à l'impact organisationnel des TI ", Tome 1, EUE, 2010.

MAKHTAR Diop, «L'Afrique, futur royaume de l'innovation ? » in Tribune du 20 octobre 2017.

MOODLEY Sagren, Perspectives de 1'E- business pour le secteur sud-africain des pièces automobiles page 75 in GOLDSTEIN Andrea et O'CONNOR David (éditeur), Commerce électronique et Développement, Etudes du centre de développement, OCDE, 2002.

OCDE (1991), La dimension économique des normes en matière de technologies de l'information, politiques d'information, d'informatique et de communications $n^{\circ} 25$, Paris.

PALVIA Shailendra C. Jain , PALVIA Prashant C. and ZIGLI Ronald M. (1992), The Global Issues of Information Technology Management, Idea Group Publishing, Harrisburg.

PALVIA Prashant, PALVIA Shailendra and ZIGLI Ronald M., Models and requirements for using Strategic Information systems in Developing nations, in International Journal of Information Management, volume 10, issue 2, June 1990, pages 117-126.

PATEYRON E. (1994), Le management stratégique de l'information, Economica Gestion Poche, Paris.

PATEYRON E. et SALMON R. (1996), Les nouvelles technologies de l'information et l'entreprise, Economica Gestion Poche, Paris.

TREMBLAY Christian (1992), L'informatique du décideur : pour intégrer l'informatique à la stratégie de l'entreprise, Marabout, Paris.

SEGUIN F. et J.F. CHANLAT J.F. (1983), L'analyse des organisations, une anthologie sociologique, Tome I Les théories de l'organisation, Gaëtan Morin éditeur, Paris.

WALLISER Bernard, Systèmes et Modèles : Introduction critique à l'analyse des systèmes, Editions du Seuil, Paris, 1977.

WILKIN Luc (1986), Informatique et Organisations, Actes du colloque de Nivelles : L'ordinateur, L'homme et L'organisation $2^{\text {ème }}$ partie, PUB.

WILKIN Luc, (1999) Principes généraux d'organisation et de gestion, 2ième édition, PUB

WISEMAN C., (1985) Strategy and computers :Information systems as competitive weapons, Homewood, IL : Dow Jones-Irwin, pour l'édition française L'informatique stratégique : nouvel atout de la compétitivité, Edition d'organisation, Paris, 1987.

ZIGLI, R.M., Rank order of African MIS issues based on personal interviews with selected Information systems executives, Working paper, The Citadel, 1990, Charleston. 
